\title{
Neutrino Halos in Clusters of Galaxies and their Weak Lensing Signature
}

\section{Francisco Villaescusa-Navarro, ${ }^{a}$ Jordi Miralda-Escudé, ${ }^{b, c}$ Carlos Peña-Garay, ${ }^{a}$ Vicent Quilis ${ }^{d}$}

\author{
${ }^{a}$ IFIC, Universidad de Valencia-CSIC, Valencia \\ ${ }^{b}$ Institució Catalana de Recerca i Estudis Avançats, Barcelona \\ ${ }^{c}$ Institut de Ciències del Cosmos (UB-IEEC), Barcelona \\ ${ }^{d}$ Departamento de Astronomia y Astrofisica, Universidad de Valencia, Valencia \\ E-mail: villa@ific.uv.es,miralda@icc.ub.es,penya@ific.uv.es, vicent.quilis@uv.es
}

\begin{abstract}
We study whether non-linear gravitational effects of relic neutrinos on the development of clustering and large-scale structure may be observable by weak gravitational lensing. We compute the density profile of relic massive neutrinos in a spherical model of a cluster of galaxies, for several neutrino mass schemes and cluster masses. Relic neutrinos add a small perturbation to the mass profile, making it more extended in the outer parts. In principle, this non-linear neutrino perturbation is detectable in an all-sky weak lensing survey such as EUCLID by averaging the shear profile of a large fraction of the visible massive clusters in the universe, or from its signature in the general weak lensing power spectrum or its cross-spectrum with galaxies. However, correctly modeling the distribution of mass in baryons and cold dark matter and suppressing any systematic errors to the accuracy required for detecting this neutrino perturbation is severely challenging.
\end{abstract}




\section{Contents}

1 Introduction 1

2 Numerical method 2

$\begin{array}{ll}2.1 \text { Mass density profile } & 2\end{array}$

2.2 Dark matter halo evolution 3

$\begin{array}{ll}2.3 & \text { Neutrino orbits } \\ \end{array}$

3 Neutrino density profiles $\quad 6$

4 Neutrino detection with weak lensing $\quad 8$

5 Conclusion $\quad 11$

\section{Introduction}

The discovery of neutrino flavour conversion of solar, atmospheric, reactor and accelerator neutrinos implies that at least two of the three light neutrinos are massive. The sum of the neutrino masses is still unknown. It is constrained from above $(\sim \mathrm{eV})$ by tritium beta decay end point data and by cosmological data, and from below $(0.05 \mathrm{eV})$ by neutrino oscillation data. The neutrino mass squared differences are precisely measured by reactor and accelerator experiments, [1]

$$
\begin{gathered}
\Delta m_{21}^{2}=(7.6 \pm 0.2) \times 10^{-5} \mathrm{eV}^{2}, \\
\Delta m_{31}^{2}=(2.4 \pm 0.1) \times 10^{-3} \mathrm{eV}^{2} .
\end{gathered}
$$

However, the neutrino mass hierarchy, or whether the two neutrinos with the smallest mass difference are heavier or lighter than the other one, is still unknown. Recent forecasts of galaxy clustering have included the neutrino mass ordering in addition to the total neutrino mass among the free model parameters that are considered, and show that future surveys should reach the sensitivity required to explore most of the allowed range of the total neutrino mass and to determine the neutrino hierarchy [2].

Neutrino masses are usually included in the list of parameters of the standard model of cosmology in the linear regime, but this has rarely been done in the nonlinear case. Massive neutrinos suppress the small scale matter power spectrum due to their large thermal velocities, making the shape of the total mass power spectrum a potential probe to neutrino masses. On scales much smaller than the free-streaming distance of neutrinos, the relative suppression is [3],

$$
\left|\frac{\Delta P(k)}{P(k)}\right| \simeq 10 \frac{\Omega_{\nu}}{\Omega_{m}}
$$

where $\Omega_{\nu} h^{2}=\left(\Sigma_{i} m_{i}\right) /(93.14 \mathrm{eV})$.

Apart from this linear effect, massive neutrinos are also expected to cluster around gravitationally collapsed dark matter haloes as their streaming velocities are reduced and become comparable to the velocity dispersions of the halos, thereby modifying the dark matter halo total mass density profile. Previous work has studied this non-linear neutrino clustering [4-9]. Here, we present a new 
calculation with updated parameters and a more realistic halo model. We also examine weak gravitational lensing as a method for an astrophysical detection of the cosmic relic neutrinos. We find that weak gravitational lensing all-sky surveys, such as the planned EUCLID mission, may detect the presence of the neutrino perturbation in the average mass density profile of clusters of galaxies, although systematic uncertainties related to the impact of baryons on the redistribution of the total mass profile are likely to be severe.

In Section 2 we describe our method for computing the relic neutrino clustering within dark matter haloes, and the results are shown in Section 3. Section 4 discusses how the effect of relic neutrino clustering within dark matter haloes can be detected by weak lensing.

We use the $\Lambda$ CDM flat model with $\Omega_{M}=0.27$ and Hubble constant $H_{0}=70 \mathrm{~km} \mathrm{~s}^{-1} \mathrm{Mpc}^{-1}$ throughout the paper, with a power spectrum normalization $\sigma_{8}=0.9$ and primordial slope $n_{s}=0.96$.

\section{Numerical method}

This section describes the method we use to compute the neutrino density profile in a spherical model of the dark matter halo.

\subsection{Mass density profile}

In this study we consider neutrinos as test particles moving in a gravitational potential determined from a spherical model of the distribution of the cold dark matter, which dominates the total mass in clusters. Our model adopts the numerical fits that have been obtained from cosmological numerical simulations of the formation of halos from cold dark matter. We calculate a density profile including the inner virialized region and the outer infall region of a halo by smoothly joining two different pieces. The first piece is the NFW profile [10], valid inside the virial radius. The second piece is obtained starting from the average initial density perturbation around a halo in a Gaussian random field, and evolving it in the non-linear regime by assuming spherical gravitational collapse without shell-crossing [11, 12]. The two pieces are joined together at an assumed epoch of observation and at a certain radius, which is determined by requiring continuity in the density profile (the derivative of the density profile is allowed to be discontinuous at the junction point).

The NFW profile has two parameters, the halo mass and its concentration parameter, and is given by

$$
\rho_{N F W}(r)=\frac{\rho_{s}}{\left(r / r_{s}\right)\left(1+r / r_{s}\right)^{2}},
$$

where the concentration parameter is $c=r_{v} / r_{s}$, and the virial radius $r_{v}$ is obtained from the halo mass as

$$
M=\frac{4 \pi}{3} \rho_{c} \Delta_{c} r_{v}^{3} .
$$

Here, $\rho_{c}$ is the critical density of the universe at redshift $\mathrm{z}$, and $\Delta_{c}$ is the halo mean density within the virial radius in units of the critical density, which for a flat universe with a cosmological constant is given by [13]:

$$
\begin{aligned}
\Delta_{c} & =18 \pi^{2}+82 x-39 x^{2}, \\
x & =\Omega(z)-1, \\
\Omega(z) & =\frac{\Omega_{m}(1+z)^{3}}{\Omega_{m}(1+z)^{3}+\Omega_{\Lambda}} .
\end{aligned}
$$


The NFW profile is a fit to the density profile of the halo obtained in numerical simulations for the virialized region. Outside this region, we use instead a density profile obtained from the mean mass distribution around any mass concentration, in a Gaussian field with power spectrum $P(k)$ [12]. Let the rms mass fluctuation within a sphere of radius $r$ be $\sigma_{M}(r)$. The average linear overdensity $\bar{\delta}_{2}=\nu_{2} \sigma_{M}\left(r_{2}\right)$ within a radius $r_{2}$, under the condition that the mean linear overdensity within the smaller radius $r_{1}$ is equal to $\bar{\delta}_{1}=\nu_{1} \sigma_{M}\left(r_{1}\right)$, can be calculated as $\nu_{2}=\gamma_{12} \nu_{1}$, where

$$
\gamma_{12}=\frac{9}{2 \pi^{2} \sigma_{M}\left(r_{1}\right) \sigma_{M}\left(r_{2}\right)} \int_{0}^{\infty} d k P(k)\left[\frac{j_{1}\left(k r_{1}\right)}{r_{1}}\right]\left[\frac{j_{1}\left(k r_{2}\right)}{r_{2}}\right],
$$

where $j_{1}$ is the spherical Bessel function. We use this equation to obtain the average linear overdensity profile around a halo.

The outer halo density profile beyond a certain radius $r_{f 0}$, which is to be determined by a matching condition that is specified below, is then calculated as follows: we start with a guessed value of $r_{f 0}$ with a mean interior overdensity $\bar{\delta}_{f 0}$ in the NFW profile. The corresponding initial radius $r_{i 0}$ is obtained from $\left(1+\bar{\delta}_{f 0}\right)=\left(r_{i 0} / r_{f 0}\right)^{3}$. Assuming the spherical collapse model with no shell-crossing (i.e., a constant interior mass), we calculate the required extrapolated linear overdensity $\bar{\delta}_{i 0}\left(r_{i 0}\right)$ to produce the final overdensity $\bar{\delta}_{f 0}\left(r_{f 0}\right)$. We then evaluate the linear mean overdensity $\bar{\delta}_{i}\left(r_{i}\right)$ at any radius $r_{i}>r_{i 0}$ with equation (2.6), using the power spectrum of Eisenstein \& Hu [14] with the parameters $\Omega_{m}=0.27, \Omega_{\Lambda}=0.73, \sigma_{8}=0.9, n_{s}=0.96$ and $h=0.7$. Finally, using again the spherical collapse model, we compute the final radius $r_{f}$ corresponding to each initial radius $r_{i}$ and its linear overdensity $\bar{\delta}_{i}\left(r_{i}\right)$. The non-linear density profile is

$$
\rho\left(r_{f}\right)=\rho_{m}\left(\frac{r_{i}}{r_{f}}\right)^{2} \frac{d r_{i}}{d r_{f}}
$$

where the mean density of the universe is $\rho_{m}=\Omega(z) \rho_{c}$.

We choose the radius $r_{f 0}$ at which the inner NFW and the outer infall density profile are matched by requiring continuity of the mass density profile. The matching point that results from this continuity requirement at a specified redshift is located in all our calculations between 1.5 and 3 times the virial radius. In Figure 1, the density profile generated using this procedure is plotted for a dark matter halo of mass $M=10^{15} h^{-1} M_{\odot}$ at $z=0.4$ (red solid line). The dashed line shows the extrapolated NFW profile beyond the matching point. At large radius, the mean density profile obviously approaches the mean density of the universe. The figure also shows the profiles of other halos at a higher redshift with the average mass of the most massive progenitor of the halo at $z=0.4$, as discussed below. The vertical dotted lines indicate the position of the virial radius of each halo.

\subsection{Dark matter halo evolution}

Modeling the orbits of relic neutrinos in a cluster halo requires the gravitational potential of the halo to be specified as a function of time. To obtain a realistic model for a typical halo, we use the results of [15] to obtain a mass of the halo as a function of time over its entire history of accretion. Obviously, there is a large dispersion in the accretion history of a halo and therefore in the evolution of its potential well, but we take an average history for the most massive progenitor as a typical case to calculate the orbits of the neutrinos in our spherical model.

We use the empirical formula of [15], obtained from a numerical fit to the results of N-body cosmological simulations, to calculate the mass and concentration parameter of the most massive progenitor of our halo of mass $M_{f}$ at the final redshift $z_{f}$, for each earlier epoch at redshift $z>z_{f}$. This is done for 100 values of the redshift $z$, distributed logarithmically between $z=z_{f}$ and $z=10$. 


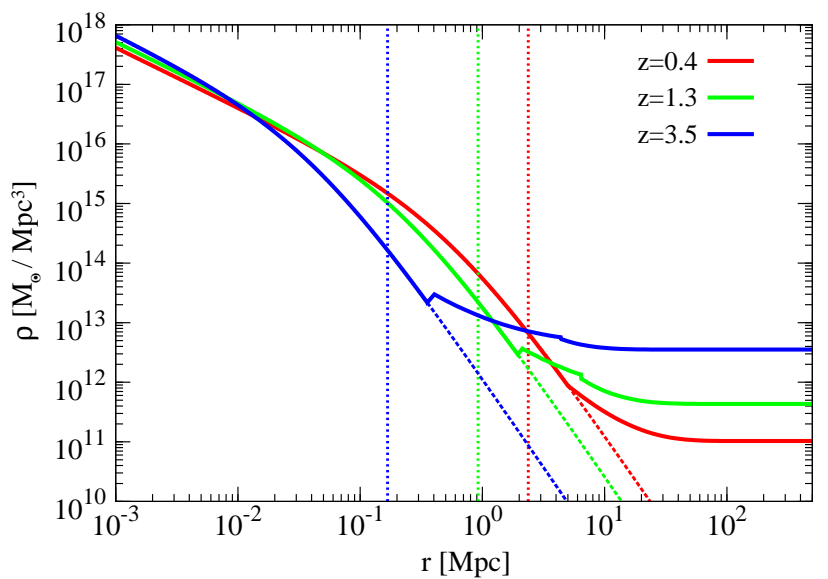

Figure 1. Red solid line shows the adopted mean dark matter halo density profile at redshift $z=0.4$ for $M_{\Delta}=10^{15} h^{-1} M_{\odot}$. The inner profile is the NFW model, matched with the outer profile computed from the average spherical perturbation around a halo evolved according to spherical collapse. The dashed line shows the extrapolation of the NFW model beyond the matching point. The green and blue lines show the density profile of the most massive progenitor at redshifts $z=1.3$ and $z=3.5$, respectively, with masses $\left(M_{\Delta}\right) 2.1 \times 10^{14} h^{-1} M_{\odot}$ and $8.5 \times 10^{12} h^{-1} M_{\odot}$. The density profiles of these halos are not continuous at the matching point, and their outer profile is determined by mass conservation as required for assembling the halo at $z=0.4$. The vertical dotted lines indicate the position of the virial radius for the three halos. The matching point chosen for continuity generally occurs around twice the virial radius.

As this work was being carried out, we initially computed the density profile of the progenitor halo of mass $M_{h}(z)$ at each redshift $z$ with the same method as for the final halo at redshift $z_{f}$, choosing a matching radius and requiring continuity with the average external density profile. However, this method does not conserve the total mass because it does not take into account the requirement that the mass $M_{f}$ that is assembled into the final halo at $z_{f}$ must be present in the external region around the progenitor halos within the shell that will finally collapse onto the halo at redshift $z_{f}$. In other words, the density profile around a progenitor halo of mass $M_{h}(z)$ is not equal to the average one as obtained from equation (2.6), but is modified by the condition that a halo of mass $M_{f}$ must be assembled at redshift $z_{f}$. Therefore, the density profile of the progenitor halo is computed by fixing the matching point to the same fixed multiple of the virial radius $r_{v}$ as for the final halo at $z_{f}, r_{f 0}(z)=r_{f 0}\left(z_{f}\right) \times r_{v}(z) / r_{v}\left(z_{f}\right)$, and tracing back in time the position of each spherical shell around the halo. At each step in redshift (backwards in time), the progenitor halo decreases its mass within $r_{f 0}(z)$ by an amount $\delta M$, and a new spherical shell is added with mass $\delta M$ with a radius equal to $r_{f 0}(z)$. All the spherical shells are traced back in time using the spherical collapse model with no shell-crossing. This results in the density profiles shown in Figure 1 for two examples of the progenitor halos, at $z=1.3$ (with mass $M=2.1 \times 10^{14} h^{-1} M_{\odot}$ ) and at $z=3.5$ (with mass $M=8.5 \times 10^{12} h^{-1} M_{\odot}$ ). The density profile is no longer continuous at the matching point, but this does not cause any problem.

We have found that correctly computing the evolution of the conserved external mass distribution around the halo of a cluster is important: if one uses instead the mean density profile around a halo progenitor, the final result for the neutrino density can be underestimated by more than a factor of two.

The evolving potential of the halo is computed by interpolation from the mass profiles calculated 
at 100 values of the redshift $z$, as the orbits of test particles representing the neutrinos are integrated.

\subsection{Neutrino orbits}

The initial phase space distribution of neutrinos is determined by their state of thermal equilibrium reached in the early universe with the primordial plasma, i.e., the Fermi-Dirac distribution for highly relativistic particles,

$$
f(p) d p=\frac{8 \pi}{(2 \pi \hbar)^{3}} \frac{p^{2} d p}{e^{p / T}+1} .
$$

The neutrino temperature, evolving as $T=T_{0}(1+z)$, is related to the photon temperature $T_{\gamma 0}$ as $T_{0}=(4 / 11)^{1 / 3} T_{\gamma 0} \approx 1.9 \mathrm{~K}$.

The orbits followed by neutrinos in our time-dependent spherical potential depend on three orbital parameters: the initial radius and momentum, and the angular momentum of the neutrino. To compute the neutrino density profile, this three-parameter space of neutrino orbits needs to be sampled densely enough to compute their average spatial distribution as a function of time. For this purpose, we divide the initial radius, momentum and angular momentum into several bins, and compute a neutrino orbit for each binned value of the three variables, starting the orbits at $z_{i}=10$. This three-dimensional phase-space grid is constructed taking particular care to resolve the particles reaching close to the center of the halo, which are at small initial radius or small angular momentum.

The grid is constructed using 10000 bins in radius from 0 to $r_{\text {max }}$, distributed as $r_{i}=r_{\max }(i / 10000)^{2}$, where $r_{\max }$ is large enough to ensure convergence of the final neutrino density profile out to a final radius of at least $30 \mathrm{Mpc}$. Momentum bins are similarly set by $p_{j}=p_{\max }(j / 500)^{2}$, with 500 bins, where $p_{\max }=0.005\left(1+z_{i}\right) \mathrm{eV}$, sufficient to sample particles out to the largest momenta making any significant contribution. Finally, the angular momentum is sampled from 0 to $L_{\max }=r_{i} p_{j}$ using 200 bins distributed as

$$
\left\{\begin{array}{r}
\theta_{k}=\left(\frac{\pi}{2}\right)\left(\frac{k}{100}\right)^{\alpha}(k \leqslant 100), \\
\theta_{k}=\pi-\left(\frac{\pi}{2}\right)\left(\frac{k-100}{100}\right)^{\alpha}(k>100),
\end{array}\right.
$$

where $\theta_{k}$ is the angle subtended between the initial momentum and radius of the particle. Here, $\alpha$ is a parameter to control the sampling of particles with low angular momentum, which are responsible for the shape of the density profile in the inner parts. Typically, it ranges between 1.5 to 5 depending on both neutrino and dark matter halo mass. At each three-dimensional bin, neutrino orbits are computed by solving the equation

$$
\frac{d^{2} r}{d t^{2}}-\frac{L^{2}}{r^{3}}=-\frac{\partial \phi(r, t)}{\partial r},
$$

where $L$ is the conserved angular momentum per unit of mass and $\phi$ is the time-dependent Newtonian gravitational potential, computed from the dark matter density profile specified in Section 2.1. The contribution of each neutrino particle to the final neutrino density profile as a function of time is counted as a spherical shell of radius $r(t)$ and mass proportional to the weight of the bin at radius $r_{i}$, momentum $p_{j}$ and angle $\theta_{k}$ in the phase space distribution,

$$
m_{p}^{i, j, k} \propto \int_{r_{i}}^{r_{i+1}} r^{2} d r \int_{p_{j}}^{p_{j+1}} \frac{p^{2} d p}{e^{p / T_{\nu}(z)}+1} \times\left\|\cos \theta_{k+1}-\cos \theta_{k}\right\| .
$$

The final neutrino density profile is obtained by adding the mass of all interior shells at any radius and time. Equation (2.10) is solved for each particle with a Runge-Kutta fourth-order integrator with variable stepsize. 

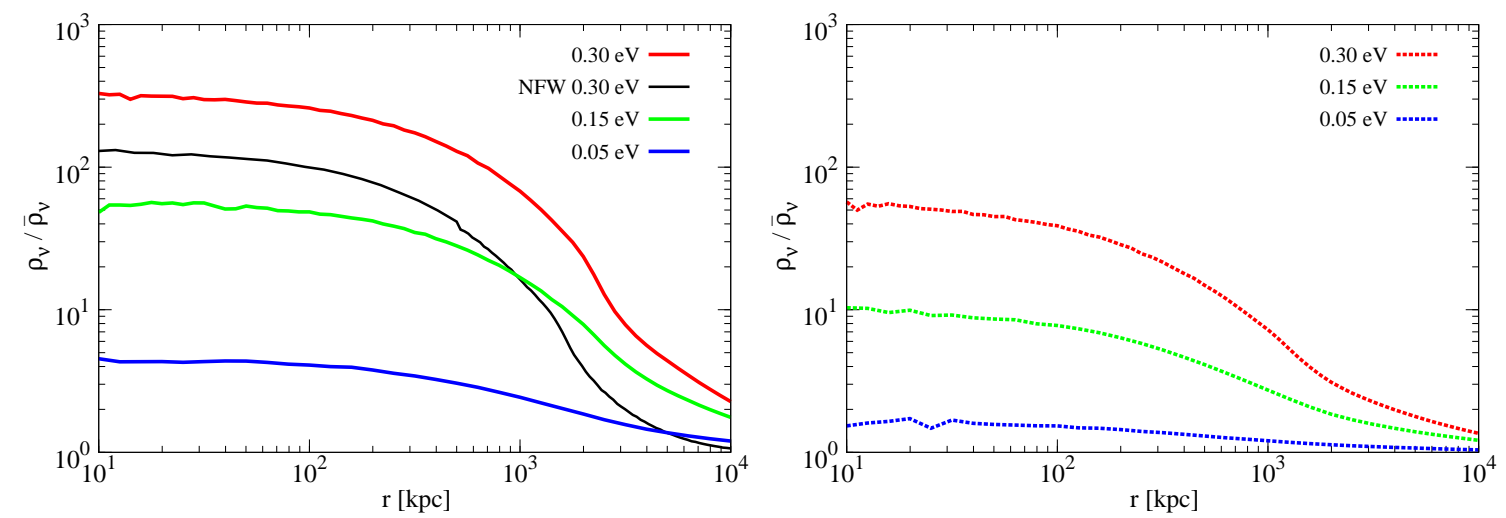

Figure 2. Neutrino density profiles at $z=0.4$, shown as the ratio to the mean neutrino density, for a halo mass of $10^{15} h^{-1} M_{\odot}$ (left panel) and $10^{14} h^{-1} M_{\odot}$ (right panel), and for the indicated neutrino masses. The case where the NFW profile is used at all radii (with a suppressed density at large radius, see Figure 1) is shown for one case in the left panel as a black line.

\section{Neutrino density profiles}

We now present the results for the spherical neutrino density profiles. We will discuss four neutrino mass schemes: a) three neutrinos with $m=0.3 \mathrm{eV}$ (labelled $0.3 \mathrm{eV}$ ), b) three neutrinos with $\mathrm{m}=0.15$ $\mathrm{eV}$ (labelled $0.15 \mathrm{eV}$ ), c) two neutrinos with $0.05 \mathrm{eV}$ and one massless neutrino (labelled IH $0.05 \mathrm{eV}$ ) and d) one neutrino with $0.05 \mathrm{eV}$ and two massless neutrinos (labelled $\mathrm{NH} 0.05 \mathrm{eV}$ ). We neglect the mass squared differences in schemes a and $\mathrm{b}$ and the small mass squared difference in schemes $\mathrm{c}$ and d. This approximation is justified because the masses of the neutrinos that we consider to have equal mass differ by less than $1 \%$ (scheme a), 5\% (scheme b), and 2\% (scheme c). In scheme $\mathrm{c}$ and d, the neutrinos that we neglect have masses smaller than $0.01 \mathrm{eV}$, and as we shall see their contribution to the total neutrino mass profile is indeed negligible. With this approximation, neutrino density profiles need to be computed only for masses of $0.3,0.15$ and $0.05 \mathrm{eV}$.

Neutrino density profiles are shown at $z=0.4$ in Figure 2 in units of the mean cosmic neutrino density, for halos of mass $10^{15} h^{-1} M_{\odot}$ (left) and $10^{14} h^{-1} M_{\odot}$ (right), and for neutrino masses of $0.05,0.15$ and $0.3 \mathrm{eV}$. The neutrino overdensity increases with both neutrino mass and halo mass, as the ratio of the halo velocity dispersion to the neutrino thermal velocities increases. The size of the core of the neutrino distribution decreases rapidly with this ratio owing to phase space density conservation. The random oscillations at small radius are due to numerical noise arising from the number of particles representing spherical shells in our simulation.

The left panel also shows, for a neutrino mass of $0.3 \mathrm{eV}$, the case of the NFW mass profile extended over all radii. This results in a reduced density, as seen in Figure 1. The reduction of the depth of the potential well in this model reduces the neutrino density.

In Figure 3, the ratio of the neutrino to the dark matter mass density profile, $\rho_{\nu} / \rho_{D M}$, is plotted for a halo of mass $10^{15} h^{-1} M_{\odot}$ at $z=0.4$, for neutrino masses of $0.3,0.15$ and $0.05 \mathrm{eV}$. Schemes a and $b$ are used for the two heavier masses (i.e., the red and green curves show the density computed for one neutrino family multiplied by 3), and schemes $\mathrm{c}$ and for the lighter mass (the cyan curve is for one neutrino family, and the blue curve is for two). A change of slope occurs at a radius close to $5 \mathrm{Mpc}$, due to the change of slope in the mass density profile at the matching point between the NFW and the outer infall model of the average density perturbation. Near a radius of $2 \mathrm{Mpc}$, a feature 


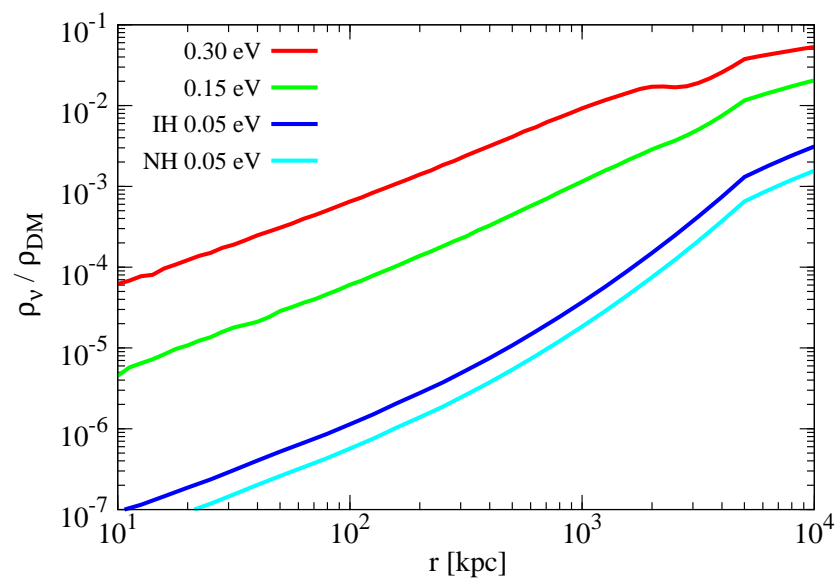

Figure 3. The neutrino to dark matter density profiles ratio at $z=0.4$ for the indicated neutrino masses, in a halo of mass of $10^{15} h^{-1} M_{\odot}$.

is present that is particularly strong for the largest neutrino mass and becomes weak as the neutrino mass is decreased. This is the result of a caustic, a special feature of spherical collapse. For perfectly cold particles, a true caustic (where the density becomes formally infinite) would appear at this radius, at which the single infalling stream of particles outside the caustic changes to a superposition of three streams inside the caustic, owing to the particles that are turning around in their first orbit after going through the halo center. The caustic is increasingly smoothed out for neutrinos as their primordial velocity dispersion increases (i.e., the neutrino mass decreases), or as the halo mass decreases. In practice, this caustic feature is present only in a spherically symmetric system. Real clusters collapsing from random initial density perturbations have caustics that are highly irregular and occur at variable radii, influenced by their internal substructure and non-sphericity, and which are largely washed out when averaging over many clusters (see, e.g., [16, 17]). Note also that a caustic should of course also be present in the Cold Dark Matter in a spherical model, which we are not taking into account here because we are using a simple analytic model for the mass profile. The Cold Dark Matter would have its caustic washed out only by the effects of substructure and non-sphericity, while the neutrino caustic is further washed out by the initial thermal velocities.

Comparing our calculations with previously published results, we find that we reproduce the results by [5] when using their dark matter halo (NFW) density profile and halo evolution model, but we do not reproduce those of [4] (see [5] for a discussion of this difference). As we have shown, the NFW halo profile extrapolated to large radius that is used by [5] underestimates the neutrino contribution to the profile at large radius. Our model also improves that of [5] on the cluster evolution, by including the mean redshift dependence of the halo progenitor mass and concentration parameter, instead of a constant halo mass during the accretion history used in [5], and by computing also the mean spherical evolution of the density profile external to the halo.

Finally, in Figure 4 we plot the neutrino surface overdensity, which is important for our lensing calculations in the next section,

$$
\Sigma_{\nu}(R)=\int_{-\infty}^{\infty}\left[\rho_{\nu}(r)-\bar{\rho}_{\nu}\right] d x
$$

where $r^{2}=x^{2}+R^{2}, R$ is the projected radius on the sky and $x$ is the dummy variable for integration along the line-of-sight, and $\bar{\rho}_{\nu}$ is the mean neutrino density, for the same cases of neutrino mass, and 

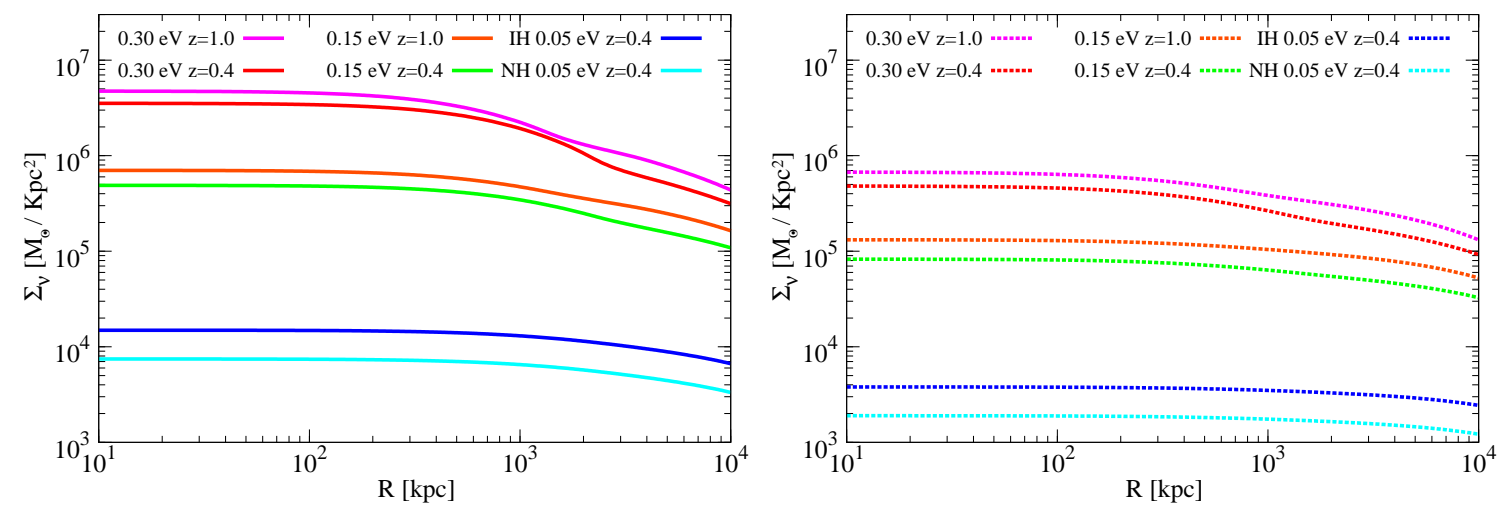

Figure 4. Neutrino surface over-density profiles for neutrinos with the indicated masses, at $z=0.4$ and $z=1$. Left panel is for halo mass $10^{15} h^{-1} M_{\odot}$, and right panel for $10^{14} h^{-1} M_{\odot}$.

at redshifts $z=0.4$ and $z=1$. The left panel is for a halo mass $10^{15} h^{-1} M_{\odot}$, and the right panel for $10^{14} h^{-1} M_{\odot}$. The same main effect is clearly seen as previously: the core radius of the neutrinos is reduced, and their central overdensity increases, as the halo mass or neutrino mass increases.

\section{Neutrino detection with weak lensing}

We now consider the possibility of detecting the perturbation caused by neutrinos on the radial density profile of a cluster using weak gravitational lensing. In this section we consider the idealized case where weak lensing can be measured for a large number of clusters with a perfectly known selection function, with statistical errors declining as the square root of the number of clusters.

We summarize first the basic concepts of weak gravitational lensing. The distortion of images behind an extended gravitational lens is determined by the surface density of the lens at every point in projection on the sky, $\Sigma$. The convergence is $\kappa=\Sigma / \Sigma_{\text {crit }}$, where $\Sigma_{\text {crit }}$ is the critical surface density, which depends on the angular diameter distances to the lens $\left(D_{l}\right)$, to the source $\left(D_{s}\right)$, and from the lens to the source $\left(D_{l s}\right)$ :

$$
\Sigma_{c r i t}=\frac{c^{2} D_{s}}{4 \pi G D_{l s} D_{l}}
$$

In general, a spherical source acquires an elliptical shape after being lensed, with axis ratio $(1-\kappa-$ $\gamma) /(1-\kappa+\gamma)$, where $\gamma$ is the shear (for reviews see, e.g., $[18,19])$. In a spherical lens, the shear is given by

$$
\gamma(R)=\bar{\kappa}(R)-\kappa(R),
$$

where $\bar{\kappa}(R)$ is the average convergence within a projected radius $R$,

$$
\bar{\kappa}(R)=\frac{2}{R^{2}} \int_{0}^{R} d R^{\prime} R^{\prime} \kappa\left(R^{\prime}\right) .
$$

The weak lensing limit is the case when $\kappa \ll 1$ and $\gamma \ll 1$, in which case the ellipticity acquired by the source is $\epsilon \simeq 2 \gamma$.

For an arbitrary mass distribution without spherical symmetry, we can choose any center we may wish and consider the values of the convergence and shear averaged on circles of radius $R$ around the chosen center. The averaged quantity $\bar{\kappa}(R)$ is also obtained by averaging the convergence 
within a radius $R$. Equation (4.2) is then just as valid for an arbitrary mass distribution, provided that we define $\kappa(R)$ and $\gamma(R)$ by averaging over circles of radius $R$ (or, in other words, circularly rotating the lens around the chosen center and averaging over all possible angles of rotation).

The quantity $\gamma(R)$ is the one we can directly measure from the shapes of the lensed galaxies, and the density profile of the cluster lens can be reconstructed by the use of inversion methods [20]. A very useful particular case is obtained by considering the integral

$$
\int_{R_{1}}^{R_{2}} \frac{d R}{R} 2 \gamma(R)=\bar{\kappa}\left(R_{1}\right)-\bar{\kappa}\left(R_{2}\right) \equiv C_{12} .
$$

This equality is easily verified from equations (4.2) and (4.3). Hence, we can measure differences in the projected mass at two different radii $R_{1}$ and $R_{2}$, from the directly observable shear in the annulus between the two radii.

The shear cannot be measured exactly because the sources have random ellipticities with dispersion $\sigma_{e}$. If the sources have a number density $n$ (considering them to be all at the same redshift for simplicity), the error in the measurement of the average $\gamma$ in an annulus of radius $R$ and width $\Delta R$ is

$$
\sigma_{\gamma}=\frac{\sigma_{e}}{2}(2 \pi R n \Delta R)^{-1 / 2} .
$$

The error in the quantity $C_{12}$ is then given by

$$
\sigma_{C}=\frac{\sigma_{e}}{2 \sqrt{\pi n R_{1}^{2}} \sqrt{1-R_{1}^{2} / R_{2}^{2}}} .
$$

The mean value of $C_{12}$ averaged over a large sample of clusters depends on the mass distribution of the clusters and any cosmological parameters that affect the average halo density profiles. Ideally, if a sample of clusters is selected in a perfectly controlled way, one can predict their mean density profile and the function $C_{12}$. The density profile is affected by neutrinos, and if all other physical factors and selection effects influencing the mean density profile are correctly known and taken into account, the presence of neutrinos may be detected from the observed shape of the cluster shear profile using weak lensing.

As a specific example, we consider the case $R_{2}=f R_{1}$, where $f$ is a constant that we fix to $f=2$. In Figure 5, the function $C_{f}(R) \equiv \bar{\kappa}(R)-\bar{\kappa}(f R)$ is plotted for four cases, with cluster masses of $10^{15} h^{-1} M_{\odot}$ and $10^{14} h^{-1} M_{\odot}$ at $z=0.4$, and masses $10^{15} h^{-1} M_{\odot}$ and $10^{14} h^{-1} M_{\odot}$ at $z=1$. The sources are assumed to lie all at $z_{s}=1.5$.

The effect of neutrinos is to modify the observable function $C_{f}(R)$ by a fractional amount $\Delta C_{f} / C_{f}$, where $\Delta C_{f}$ is calculated for the neutrino density profile in the same way as $C_{f}$ for the total mass profile. This ratio is plotted in Figure 6 for various neutrino masses and for two different dark matter halo masses. The ratio increases with neutrino mass and grows with radius because the neutrino density profile is extended. For the cases that are shown, the fractional weak lensing effect of neutrinos does not change much with halo mass, although the observable effect, $\Delta C_{f}$, obviously increases with halo mass, as shown in Figure 7.

The predicted neutrino effect is very small, and it might only be observable as a perturbation in the mean cluster shear profile by averaging over many clusters. The requirement for detecting the neutrino effect can be estimated by considering the cluster in Figure 5 with a halo mass $10^{15} h^{-1} M_{\odot}$ at $z=0.4$, and sources with number density $n=30 \operatorname{arcmin}^{-2}$ located at $z=1.5$ and at the radius $r_{1}=2 \mathrm{Mpc}$ (corresponding to 6 arc minutes). The 1- $\sigma$ error on $C_{f}$ is $\sigma_{C}=0.002$ if we use $\sigma_{e}=0.2$, while its value is $C_{f}\left(r_{1}\right) \simeq 0.01$. Therefore, we may reach an accuracy of $20 \%$ on the measurement of $C_{f}$ with a single cluster. To be able to measure the difference between different neutrino models 


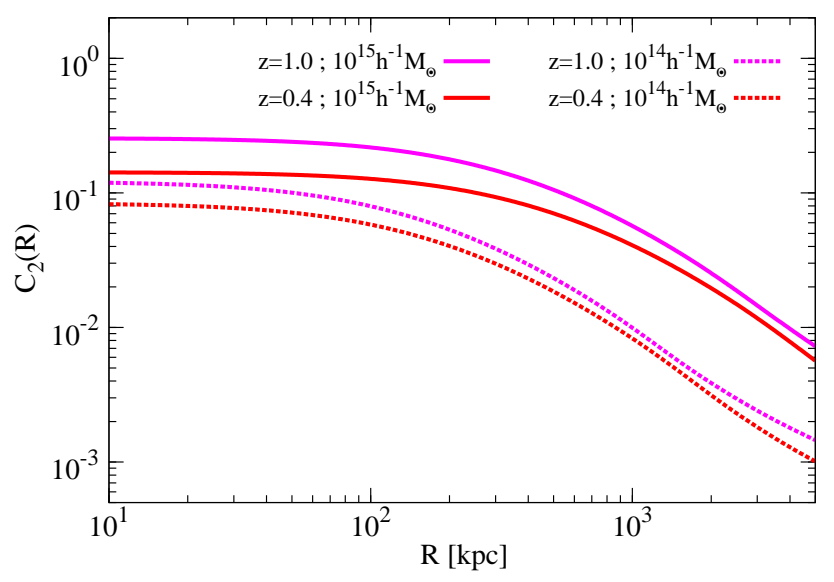

Figure 5. The function $C_{f}(R)$, with $f=2$, for four dark matter halos with the masses and redshifts indicated.
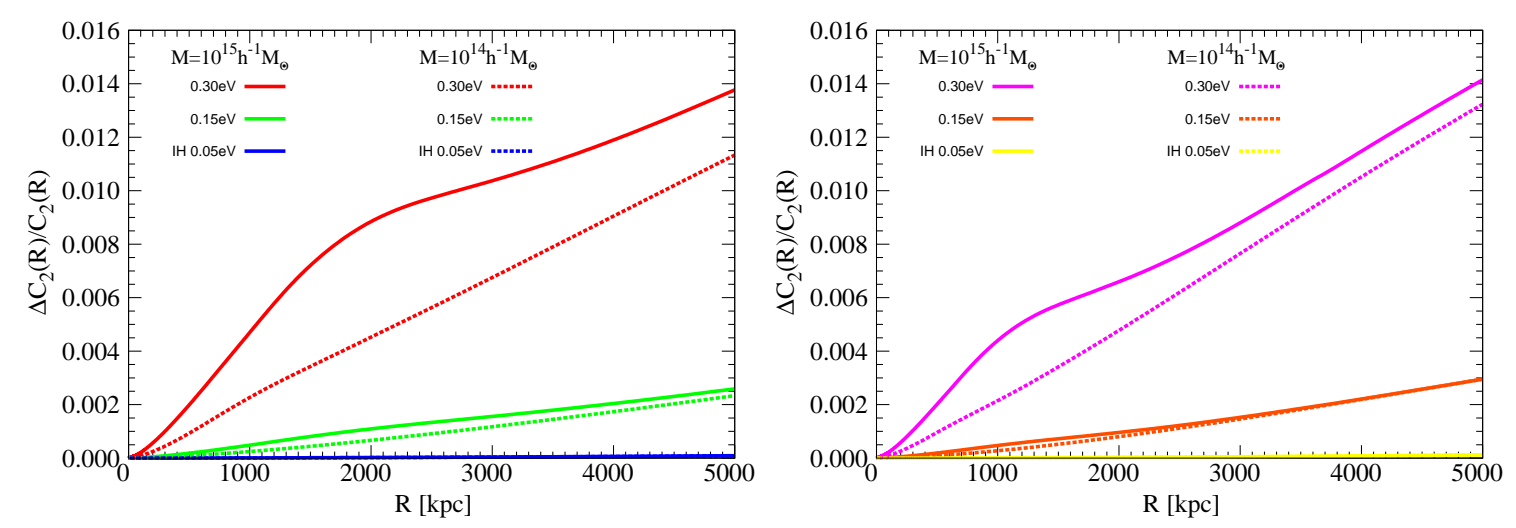

Figure 6. Fractional neutrino perturbation on the weak lensing profile, $\Delta C_{f} / C_{f}$, versus radius, for the cluster masses and neutrino masses indicated. The left panel is for clusters at $z=0.4$, and the right panel at $z=1$.

of $\Delta C_{f} / C_{f}<0.01$, as expected from Figure 6 for a neutrino mass of $0.3 \mathrm{eV}$, one would need to average the measurement of the shear over 10000 clusters to obtain a 5- $\sigma$ result.

This is approximately the number of massive clusters that might be observed in an all-sky weak lensing survey of sufficient depth. Therefore, the measurement of the neutrino perturbation on the mean density profile of clusters is extremely difficult. Apart from the need to observe a very large number of clusters to reduce the statistical error, systematic uncertainties would in practice be even more difficult to resolve. The theoretical prediction for the precise density profile in the absence of neutrinos needs to be sufficiently reliable, but this profile is affected by several variables that may be hard to control: the precise selection function of clusters of different masses and different spatial orientations and projection effects would need to be accurately modeled using numerical simulations of structure formation, and the contribution from baryons would be subject to uncertainties related to radiative cooling, galactic winds, and generally the way that galaxy formation may alter the mass distribution. The detection of the gravitational effect of neutrinos from lensing seems therefore a very difficult challenge.

The calculation presented in this paper should be considered only as an illustrative case. In 

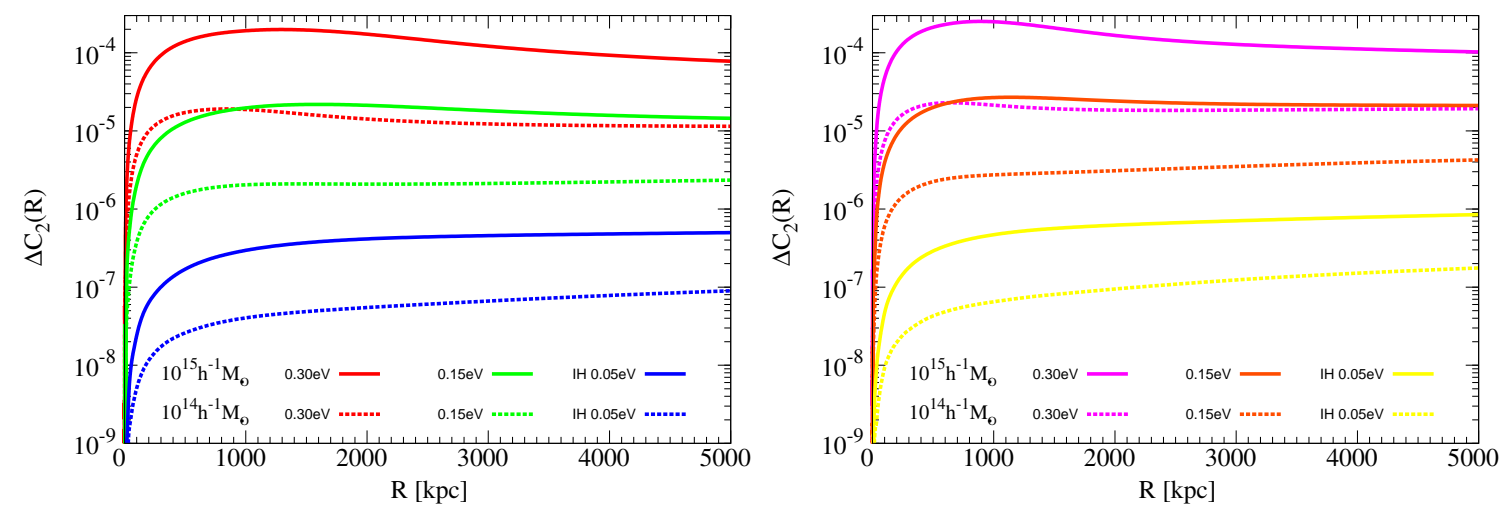

Figure 7. Neutrino perturbation on the weak lensing profile, $\Delta C_{f}$, versus radius, for the same cluster and neutrino masses as in Figure 6. Left panel is for halos at $z=0.4$ and right panel at $z=1$.

practice, a better approach for attempting to measure the clustering effects of neutrinos may be to examine directly the power spectrum and bispectrum of the weak lensing shear over the whole field, thereby avoiding the issue of selection effects in a cluster sample. However, this would necessarily average out the effects of neutrinos in the regions where they are strongest, in massive clusters of galaxies. The cross-correlation of lensing shear with massive galaxies or diffuse X-ray emission that are associated with clusters would also likely be subject to similar uncertainties arising from the precise selection function.

\section{Conclusion}

We have presented the clustering of relic neutrinos around spherical dark matter for various illustrative cases. Neutrinos produce an extended distribution of mass with a large core determined by their primordial thermal velocities, which cause a perturbation on the total density profile. The nonlinear collapse of neutrinos in massive clusters should modify their impact on the overall mass power spectrum of fluctuations calculated in the linear regime.

The presence of the neutrino perturbation in the average mass density profile of clusters of galaxies using weak gravitational lensing would constitute a remarkable astrophysical detection of the cosmic relic neutrinos, which cannot be detected by any other known method, except for their linear contribution to the total matter power spectrum. However, this measurement is a very difficult one owing to the small contribution that neutrinos make to the cluster mass even at very large radius. For a neutrino mass of $0.3 \mathrm{eV}$, the largest value that is compatible with current experimental constraints, the lensing shear profile of a massive cluster is affected by neutrinos roughly at the level of $1 \%$. This small signal can only be detected by averaging lensing measurements over observable clusters in a large fraction of the sky. Although this observation can be done with an all-sky weak lensing space mission, such as the planned EUCLID mission, systematic uncertainties related to the impact of physical effects such as the distribution of baryons and the precise cluster selection function would make this detection a difficult one. 


\section{Acknowledgments}

FV would like to thank Neal Dalal, Urbano França, Olga Mena and Manuel Peña for discussions and revisions of this paper. The numerical computations were carried out in the Sunnyvale cluster at the Canadian Institute for Theoretical Astrophysics (CITA). This work is supported in part by the Spanish MICINN grants FPA-2007-60323, AYA2009-09745, AYA2010-21322-C03-01, the Consolider Ingenio-2010 project CSD2007-00060 and the Generalitat Valenciana grant PROMETEO/2009/116.

\section{References}

[1] M. C. Gonzalez-Garcia, M. Maltoni and J. Salvado, JHEP 1004, 056 (2010) [arXiv:1001.4524 [hep-ph]].

[2] R. Jimenez, T. Kitching, C. Pena-Garay and L. Verde, JCAP 1005, 035 (2010) [arXiv:1003.5918 [astro-ph.CO]].

[3] J. Lesgourgues and S. Pastor, Phys. Rept. 429, 307 (2006) [arXiv:astro-ph/0603494].

[4] S. Singh and C. P. Ma, Phys. Rev. D 67, 023506 (2003) [arXiv:astro-ph/0208419].

[5] A. Ringwald and Y. Y. Y. Wong, JCAP 0412, 005 (2004) [arXiv:hep-ph/0408241].

[6] J. Brandbyge and S. Hannestad, JCAP 0905, 002 (2009) [arXiv:0812.3149 [astro-ph]].

[7] J. Brandbyge, S. Hannestad, T. Haugboelle and Y. Y. Y. Wong, JCAP 1009, 014 (2010) [arXiv:1004.4105 [astro-ph.CO]].

[8] M. Viel, M. G. Haehnelt and V. Springel, JCAP 1006, 015 (2010) [arXiv:1003.2422 [astro-ph.CO]].

[9] F. Marulli, C. Carbone, M. Viel, L. Moscardini and A. Cimatti, arXiv:1103.0278 [astro-ph.CO].

[10] J. F. Navarro, C. S. Frenk and S. D. M. White, Astrophys. J. 462, 563 (1996) [arXiv:astro-ph/9508025].

[11] J. M. Bardeen, J. R. Bond, N. Kaiser and A. S. Szalay, Astrophys. J. 304, 15 (1986).

[12] A. Loeb and D. J. Eisenstein, Astrophys. J. 448, 17 (1995) [arXiv:astro-ph/9411054].

[13] G. L. Bryan and M. L. Norman, Astrophys. J. 495, 80 (1998) [arXiv:astro-ph/9710107].

[14] D. J. Eisenstein and W. Hu, Astrophys. J. 511, 5 (1997) [arXiv:astro-ph/9710252].

[15] D. H. Zhao, Y. P. Jing, H. J. Mo and G. Boerner, Astrophys. J. 707, 354 (2009) [arXiv:0811.0828 [astro-ph]].

[16] J. A. Fillmore and P. Goldreich, Astrophys. J. 281, 1 (1984).

[17] M. Vogelsberger, S. D. M. White, R. Mohayaee and V. Springel, Mon. Not. Roy. Astron. Soc. 400, 2174 (2009) arXiv:0906.4341 [astro-ph.CO]..

[18] P. Schneider, J. Ehlers, E. E. Falco, Astronomy and Astrophysics Library, Springer (1992).

[19] R. Blandford, R. Narayan, Astrophys. J. 310, 568 (1986).

[20] N. Kaiser and G. Squires, Astrophys. J. 404, 441 (1993). 